\title{
miR-204 inhibits the osteogenic differentiation of mesenchymal stem cells by targeting bone morphogenetic protein 2
}

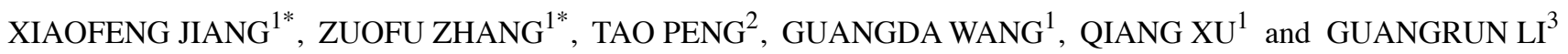 \\ ${ }^{1}$ Department of Joint Surgery, Yantai Yuhuangding Hospital, Yantai, Shandong 264000; \\ ${ }^{2}$ Department of Orthopedics, Pingdu People's Hospital, Pingdu, Shandong 266700; \\ ${ }^{3}$ Department of Spinal Surgery, Yantai Yuhuangding Hospital, Yantai, Shandong 264000, P.R. China
}

Received April 11, 2019; Accepted May 15, 2019

DOI: $10.3892 / \mathrm{mmr} .2019 .10791$

\begin{abstract}
Mesenchymal stem cells (MSCs) are used to investigate regeneration and differentiation. MicroRNA-204 (miR-204) in involved in the Runt-related transcription factor 2/alkaline phosphatase/bone morphogenic protein 2 (Runx2/ALP/BMP2) signaling pathway that regulates bone marrow mesenchymal stem cell (BMSC) differentiation; however, the mechanisms underlying the effects of miR-204 are yet to be determined. The aim of the present study was to investigate the effects of miR-204 on BMSC differentiation. BMSCs were derived from rat bone marrow. The expression levels of Runx2, ALP and BMP2 were measured via reverse transcription-quantitative polymerase chain reaction and western blot analyses following transfection of BMSCs with miR-204 agomir or BMP2 expression vector. The ability of the miR-204 gene to directly bind BMP2 mRNA was assessed using dual-luciferase assays. Ossification was measured via alizarin red stain assays. It was observed that the expression levels of Runx 2 and ALP increased over time, whereas those of miR-204 decreased; additionally, miR-204 agomir upregulation inhibited the expression of Runx2, ALP and BMP2 in BMSCs. It was revealed that miR-204 directly interacted with BMP2 mRNA, and that transfection with miR-204 agomir suppressed ossification in BMSCs by targeting the BMP2/Runx2/ALP signaling pathway.
\end{abstract}

\section{Introduction}

A balance between bone formation and resorption maintains the integrity of skeleton, and such a balance is realized by

Correspondence to: Dr Guangrun Li, Department of Spinal Surgery, Yantai Yuhuangding Hospital, 20 East Yuhuangding Road, Yantai, Shandong 264000, P.R. China

E-mail: lgrun_grli@163.com

*Contributed equally

Key words: bone marrow mesenchymal stem cells, microRNA-204, bone morphogenic protein 2 , osteogenic differentiation osteoclasts and osteoblasts $(1,2)$. Osteoblast differentiation stimulates bone formation and repair (3). Therefore, osteogenic differentiation has a connection with bone disease (bone fracture and osteoporosis) and bone implantation (4-7). The differentiation of bone marrow mesenchymal stem cells (BMSCs) involves a number of signaling pathways, including the hypoxia-inducible factor $1 \alpha$ pathway, mechano-growth factor signaling, the leukemia inhibitory factor/STAT/suppressor of cytokine signaling 3 pathway and NF- $\kappa$ B signaling (8-10).

MicroRNAs (miRNAs), small non-protein coding RNAs ( $\sim 22$ nucleotides), serve important roles in the regulation of gene expression by binding to the 3'-untranslated region (3'-UTR) of target messenger RNAs (mRNAs) $(11,12)$. Located at cancer-related gene regions 9q21.1-q22.3 (13), a previous study reported that miR-204 regulated cancer cell proliferation and invasion by targeting cyclin D2 and matrix metalloproteinase-9 (14). In addition, prior evidence suggested that miR-204 overexpression inhibited osteogenic differentiation and promoted adipogenic differentiation $(14,15)$; however, the main mechanisms underlying the effects of miR-204 on BMSCs are yet to be determined. The present study investigated the role of the Runt-related transcription factor 2/alkaline phosphatase/bone morphogenic protein 2 (Runx2/ALP/BMP2) signaling pathway in the effects of miR-204 on BMSCs.

It was previously demonstrated that increased Runx 2 levels were observed in mesenchymal stem cells derived from multiple myeloma patients compared with normal MSCs, and that upregulation of Runx2 resulted in bone defects (16). ALP is a glycoprotein involved in mineral formation in bone tissue; ALP activity has a positive effect on the mineralization process for cellular cementum formation (17). Increased serum alkaline phosphatase (ALP) activity has been reported in rheumatoid arthritis (RA) (18). BMP2 belongs to the BMP family, which possess diverse biological functions during osteogenesis and osteogenic differentiation, including the maintenance of normal bone and bone regeneration (19-22). Furthermore, the BMP family regulates osteogenic differentiation $(23,24)$.

The aim of the present study was to investigate whether miR-204 affected osteoblast differentiation and the potential underlying mechanisms. The effects of miR-204 on the osteogenic differentiation of MSCs were determined by evaluating Runx2, BMP2 and ALP expression. Additionally, the regulatory target of miR-204 was explored. 


\section{Materials and methods}

Cell identification and cell culture. BMSCs were isolated from rat bone marrow. Male Sprague-Dawley (SD) rats aged 4 weeks were purchased from Shangdong Laboratory Animal Center (Jinan, China). The rat bone marrow tissues were extracted after the rats were sacrificed. The femur and tibia were washed with phosphate buffered saline (PBS; Gibco; Thermo Fisher Scientific, Inc.) and cut into $5 \mathrm{~mm}$ pieces, high-glucose DMEM (basic medium; Thermo Fisher Scientific, Inc.) was used to wash the bone fragments, and the solution was centrifuged at $675 \mathrm{x}$ g for $5 \mathrm{~min}$ at room temperature prior to resuspension of cells in PBS. The cells were separated using lymphocyte separation medium (Beijing Solarbio Science \& Technology Co., Ltd.) and the BMSCs were acquired. The animal study was approved by the Institutional Animal Care and Use Committee of Yantai Yuhuangding Hospital. BMSCs were seeded at $3.5 \times 10^{5}$ cells/dish in $75-\mathrm{mm}$ culture dishes (Corning Inc.) in basic medium with $10 \%$ fetal bovine serum (FBS; Gibco; Thermo Fisher Scientific, Inc.) and $100 \mathrm{U} / \mathrm{ml}$ penicillin $/ 100 \mu \mathrm{g} / \mathrm{ml}$ streptomycin (Gibco; Thermo Fisher Scientific, Inc.) in a $5 \% \mathrm{CO}_{2}$ atmosphere at $37^{\circ} \mathrm{C}$. BMSCs were subcultured when the cells reached $90 \%$ confluency. The cells were centrifuged at $750 \mathrm{x}$ g for $5 \mathrm{~min}$ at room temperature after digestion using $0.25 \%$ trypsin-EDTA (Gibco; Thermo Fisher Scientific, Inc.) at $37^{\circ} \mathrm{C}$ for $5 \mathrm{~min}$ in an incubator (Thermo Fisher Scientific, Inc.). Purified BMSCs $\left(2 \times 10^{7}\right.$ cells $\left./ \mathrm{ml}\right)$ were seeded in 6-well plates and cluster of differentiation (CD) 90-allophycocyanin (APC; 1:20; cat. no. 17-5900-42; Invitrogen; Thermo Fisher Scientific, Inc.), CD45-phycoerythrin/cyanine7 (PE/CY7; $0.1 \mathrm{mg} / \mathrm{ml}$; cat. no. 1660-17, SouthernBiotech) and CD11b/c-FITC (30 $\mu \mathrm{g} / \mathrm{ml}$, cat. no. 130-105-273; Miltenyi Biotec, Inc.) antibodies were used to stain the samples in a final volume of $100 \mu \mathrm{l}$ at $4^{\circ} \mathrm{C}$ for $20 \mathrm{~min}$. Then, BMSCs in solution were identified via flow cytometry and analyzed with CellQuest pro software version 5.1 (BD Biosciences), by detecting the absorbance at $647 \mathrm{~nm}$ for CD90-APC, $532 \mathrm{~nm}$ for CD45-PE/CY7 and $488 \mathrm{~nm}$ for CD11b/c-FITC.

Cell transfection. BMSCs were seeded in 75-mm culture dishes at $3.5 \times 10^{5}$ cells/dish and incubated for $24 \mathrm{~h}$. miR-204 agomir (5'-UUCCCUUUGUCAUCCUAUGCC-3'), miR-204 antagomir (5'-AGGCAUGGAUGACAAAGGGAA-3') and negative control (NC, 5'-CAGUACUUUUGUGUAGUA CAA-3') sequences were purchased from Guangzhou RiboBio Co., Ltd. pLVX-AcGFP-C1-BMP2 was constructed by cloning amplified BMP2 into pLVX-AcGFP-C1 (Sangon Biotech Co., Ltd.). pLVX-AcGFP-C1 was used as the negative control. Transfections were performed using Lipofectamine ${ }^{\circledR} 2000$ (Invitrogen; Thermo Fisher Scientific, Inc.). Transfection complexes were added to the medium at a final concentration of $50 \mathrm{nM}$. The transfection solution was added to BMSCs for 3,5 and 7 days at $37^{\circ} \mathrm{C}$ in a $5 \% \mathrm{CO}_{2}$ in an incubator.

Western blotting. BMSCs were transfected for 3 days. Then, the total proteins were extracted using RIPA buffer (Invitrogen; Thermo Fisher Scientific, Inc.) at $4^{\circ} \mathrm{C}$. Protein concentration were determined using the BCA method. Proteins $(20 \mu \mathrm{g} / \mathrm{lane})$ were electrophoresed on 10\% SDS-PAGE gels and transferred to polyvinylidene fluoride membranes (Thermo Fisher
Scientific, Inc.). After blocking with 5\% non-fat milk at room temperature for $2 \mathrm{~h}$, membranes were incubated overnight at $4^{\circ} \mathrm{C}$ with antibodies against Runx2 (1:1,000; cat. no. 12556; Cell Signaling Technology, Inc.), ALP (1:1,000; cat. no. ab83259; Abcam), BMP2 (1:1,000; cat. no. ab14933; Abcam) and $\beta$-actin (1:1,000; cat. no. 4970; Cell Signaling Technology, Inc.). After the membranes were washed three times in TBS- $0.5 \%$ Tween-20 (TBST), a horseradish peroxidase-conjugated secondary antibody (1:10,000; cat. no. 10285-1-AP; ProteintTech Group, Inc.) was used to incubate the membranes for 2-3 $\mathrm{h}$ at room temperature. The membranes were then washed 2-3 times in TBST solution. The blots were visualized using ECL (Thermo Fisher Scientific, Inc.). An ECL system (Amersham; GE Healthcare) was used to visualize the bands. Quantity one software version 4.6.2 (Bio-Rad Laboratories, Inc.) was used for densitometry analysis.

Bioinformatics analysis. TargetScan (http://www.targetscan. org/vert_72/) was used to predict the targets of miR-204.

Dual-luciferase assay. The BMP2-3'-UTR and mutant (mut) BMP2-3'-UTR were inserted into psi-CHECK-2 plasmids (Promega Corporation), and then BMSCs were incubated with BMP2-3'-UTR or BMP2-3'-UTR mut plasmids at $37^{\circ} \mathrm{C}$ with $5 \% \mathrm{CO}_{2}$ in an incubator for $72 \mathrm{~h}$. The total protein of BMSCs transfected with luciferase plasmids was extracted using RIPA buffer (Invitrogen; Thermo Fisher Scientific, Inc.) on ice. Prior to analysis using a luciferase reporter assay kit (BioVision, Inc.), cells $\left(2 \times 10^{5}\right.$ cells/well) were plated in 24 -well plates and were co-transfected with miR plasmids at a final concentration of $50 \mathrm{nM}$ using Lipofectamine $2000^{\circledR}$. The luciferase activity of BMSCs was measured 3 days after transfection using a luciferase reporter assay kit (BioVision, Inc.). Firefly luciferase activity was normalized with renilla luciferase activity (Promega Corporation).

Reverse transcription-quantitative polymerase chain reaction $(R T-q P C R)$. Total RNA was collected as follows: BMSCs were treated with TRIzol ${ }^{\circledR}$ (Invitrogen; Thermo Fisher Scientific, Inc.) via centrifugation at 3,000 $\mathrm{g}$ g for $10 \mathrm{~min}$ at room temperature. RT was performed to synthesize cDNA using $2 \mu \mathrm{l} \mathrm{FQ-RT}$ Primer Mix, $2 \mu 1$ 10X Fast RT Buffer, $1 \mu \mathrm{l}$ RT Enzyme Mix and RNA-Free $\mathrm{ddH}_{2} \mathrm{O}$ (to $10 \mu \mathrm{l}$; all Tiangen Biotech Co., Ltd.); RT was conducted at $42^{\circ} \mathrm{C}$ for $15 \mathrm{~min}$ and $95^{\circ} \mathrm{C}$ for $3 \mathrm{~min}$. qPCR was conducted using cDNA, forward and reverse primers, and 2X PCR Taq Master Mix (MedChemExpress LLC) under the following conditions: 40 cycles of $94^{\circ} \mathrm{C}$ for $5 \mathrm{~min}, 94^{\circ} \mathrm{C}$ for $30 \mathrm{sec}, 60^{\circ} \mathrm{C}$ for $40 \mathrm{sec}$ and $72^{\circ} \mathrm{C}$ for $50 \mathrm{sec}$.

The following primers were used for qPCR: U6, forward 5'-CTCGCTTCGGCAGCACA-3', reverse 5'-ACGCTTCAC GAATTTGCGT-3'; BMP2, forward 5'-ACCAGCATTAGC ATCACG-3', reverse 5'-AGGTCCTTGGGTTGTTTT-3'; Runx2, forward 5'-CTCGCTTCGGCAGCACA-3', reverse 5'-AACGCTTCACGAATTTGCGT-3'; ALP, forward 5'-CTG ATCAGTGTGCCCCTGCAG-3', reverse 5'-GGAGCTTGG AACGAATGTTCTG-3'; and miR-204, forward 5'-CTGATC AGTGTGCCCCTGCA-3' and reverse 5'-GGAGCTTGGAAC GAATGTTCTG-3'. U6 was used as an internal reference for qPCR, and the $2^{-\Delta \Delta C q}$ method was applied to calculate relative expression levels (25). 

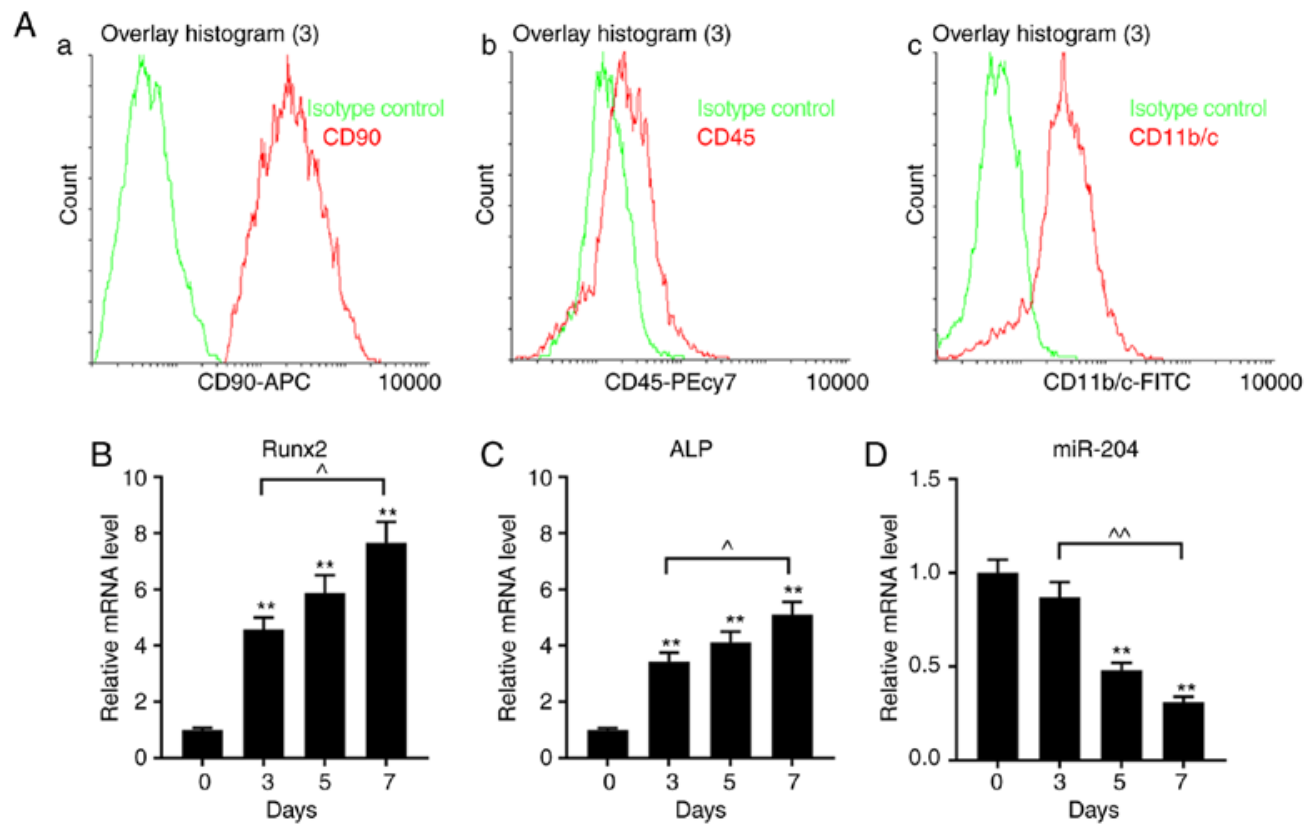

Figure 1. Properties of cultured BMSCs isolated from rat bone marrow. (A) CD expression phenotypes of BMSCs were measured using flow cytometry with (Aa) CD90-APC, (Ab) CD45-PE/CY7 and (Ac) CD11b/c-FITC. The green lines represent the isotype/negative control. (B-D) Expression of Runx2, ALP and miR-204 in BMSCs cultured for 0,3,5 and 7 days, as determined via reverse transcription-quantitative PCR analysis. Data are presented as the mean \pm standard deviation and analyzed by ANOVA followed by Tukey-Kramer multiple comparison post hoc tests. ${ }^{* *} \mathrm{P}<0.01$ vs. day 0 ; ${ }^{\wedge} \mathrm{P}<0.05$, ${ }^{\wedge} \mathrm{P}<0.01$. BMSC, bone marrow mesenchymal stem cell; miR-204, microRNA-204; CD, cluster of differentiation; ALP, alkaline phosphatase; Runx2, Runt-related transcription factor 2; APC, allophycocyanin; PE, phycoerythrin; Cy7, cyanine7.

Alizarin red stain assays. BMSCs were seeded into 24-well plates at 1,000 cells/well for $24 \mathrm{~h}$. NC, miR-204 agomir, BMP2 and miR-204 agomir + BMP2 were transfected as aforementioned when the BMSCs reached $30 \%$ confluency. After $12 \mathrm{~h}$, basic medium was replaced with culture medium containing vitamin C (50 $\mu \mathrm{g} / \mathrm{ml}$; Invitrogen; Thermo Fisher Scientific, Inc.) and $\beta$-phosphoglycerol $(10 \mathrm{mmol} / \mathrm{l}$; Invitrogen; Thermo Fisher Scientific, Inc.), and BMSCs were cultured with the aforementioned transfection reagents for a further 15 days. BMSCs were stained with $0.1 \%$ alizarin red at $37^{\circ} \mathrm{C}$ for $30 \mathrm{~min}$ following fixation with $10 \%$ glutaraldehyde for $10 \mathrm{~min}$. Images were acquired using an inverted light microscope (magnification, x100; Olympus Corporation). For each sample 5 fields were analyzed. Images were analyzed using ImageJ software version 2.0.0 (National Institutes of Health).

Statistical analysis. Statistical analysis was performed using GraphPad Prism version 6.0 (GraphPad Software, Inc.). Data were presented as the mean \pm standard deviation and all experiments were repeated at least three times. Groups were analyzed by ANOVA followed by a Tukey-Kramer post hoc multiple comparison test. $\mathrm{P}<0.05$ was considered to indicate a statistically significant difference.

\section{Results}

Expression of Runx2, ALP and miR-204 in BMSCs isolated from rats. The phenotype of BMSCs was measured using flow cytometry using CD90-APC, CD45-PE/CY7 and $\mathrm{CD} 11 \mathrm{~b} / \mathrm{c}-\mathrm{FITC}$ kits. It was revealed that the phenotype of the BMSCs was CD90+, CD45- and CD11b- (Fig. 1Aa-c). The expression levels of Runx 2 and ALP in BMSCs increased in a time-dependent manner, whereas those of miR-204 decreased (Fig. 1B-D).

Effects of miR-204 agomir on the expression levels of Runx2 and ALP in BMSCs. Transfection with miR-204 antagomir significantly inhibited the expression of miR-204 in BMSCs, whereas miR-204 agomir induced opposing effects (Fig. 2A). Cells transfected with miR-204 agomir exhibited significantly downregulated expression of Runx 2 and ALP at the mRNA and protein levels; conversely, miR-204 antagomir promoted the expression of Runx 2 and ALP (Fig. 2B-F).

Effects of miR-204 on BMP2 in BMSCs. A luciferase assay was performed to investigate the potential association between miR-204 and BMP2. Co-transfection with miR-204 + BMP2-3'-UTR resulted in significantly decreased relative luciferase activity compared with control + BMP2-3'-UTR or miR-204 + BMP2-3'-UTR mut co-transfections (Fig. 3D and E). Additionally, transfection with miR-204 agomir revealed that upregulation of miR-204 significantly decreased the expression of BMP 2 at the mRNA and protein levels compared with the NC, whereas miR-204 antagomir induced opposing effects (Fig. 3F-H).

Effects of miR-204 agomir and BMP2 on the expression of Runx2, ALP and BMP2 in BMSCs. To investigate the role of BMP2 in the effects of miR-204 on BMSCs, a BMP2 overexpression vector was used. Transfection with the BMP2 vector upregulated the expression of BMP2 at the mRNA and protein levels (Fig. 3A-C). Following transfection of BMSCs with miR-204 agomir and/or BMP2 for 3 days, the expression levels of Runx2, ALP and BMP2 

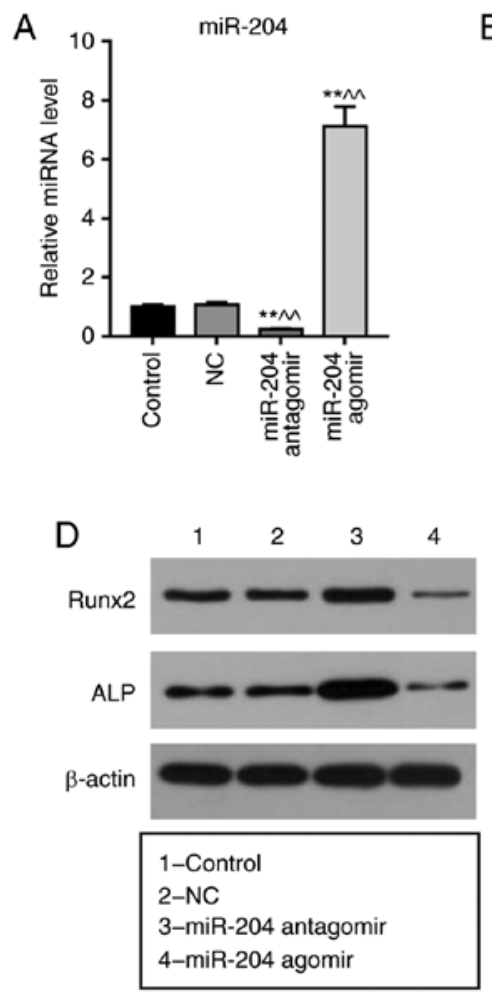
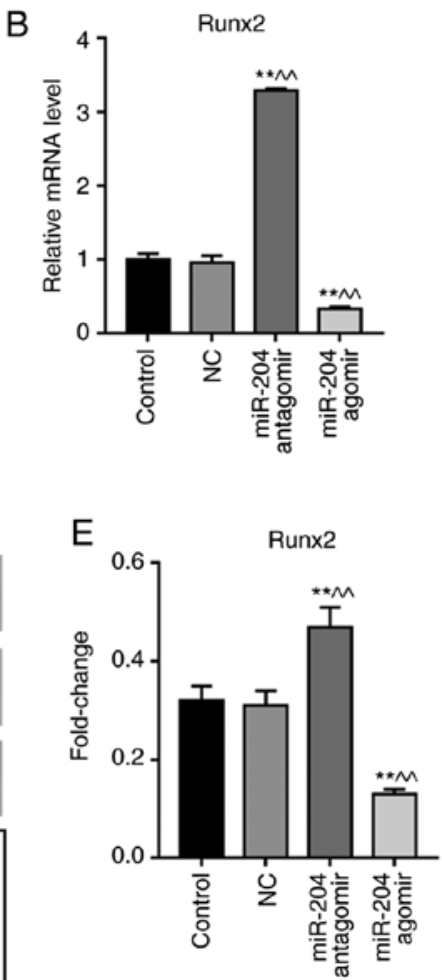
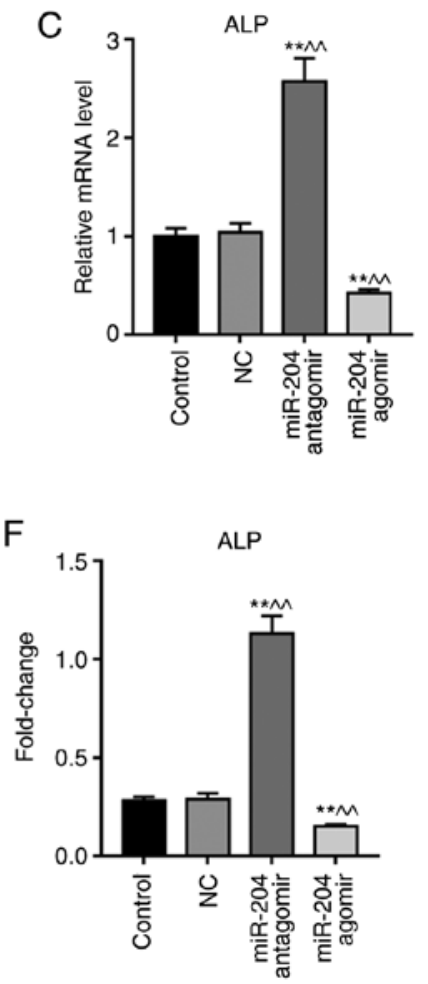

Figure 2. Effects of miR-204 on the expression levels of BMP2, Runx2 and ALP in BMSCs. BMSCs were treated with NC, miR-204 agomir or miR-204 antagomir for 3 days. $\beta$-actin and U6 were used as internal references. mRNA expression levels of (A) miR-204, (B) Runx2 and (C) ALP as determined via reverse transcription-quantitative PCR analysis. (D-F) Protein expression Runx2 and ALP as determined via western blot analyses. Data are presented as the mean \pm standard deviation and analyzed by ANOVA followed by Tukey-Kramer multiple comparison post hoc tests. ${ }^{* *} \mathrm{P}<0.01$ vs. control; ${ }^{\wedge} \mathrm{P}<0.01$ vs. NC. BMSC, bone marrow mesenchymal stem cell; miRNA/miR, microRNA; ALP, alkaline phosphatase; Runx2, Runt-related transcription factor 2; NC, negative control.

were determined via RT-qPCR and western blot analyses. It was observed that BMSCs transfected with miR-204 agomir exhibited significantly downregulated expression of BMP2, Runx 2 and ALP compared with the NC (Fig. 3I-L). BMP2 overexpression significantly upregulated the expression of Runx 2 and ALP in BMSCs compared with the NC; additionally, BMP2 overexpression significantly increased the expression levels of BMP2, Runx2 and ALP in miR-204 agomir-treated BMSCs compared with miR-204 agomir treatment alone (Fig. 3I-L).

Effects of miR-204 agomir and BMP2 on calcification in BMSCs. BMSCs were treated with NC, miR-204 agomir, miR-204 agomir + BMP2 or BMP2 for 15 days. BMSCs were stained with alizarin red. It was observed that the intensity of alizarin red staining was significantly increased in the BMP2-overexpressing group compared with in the other groups, and that the intensity in the miR-204 agomir group was significantly reduced compared with the NC (Fig. 4). The findings indicated that BMP2 promoted the calcification of BMSCs, whereas miR-204 agomir inhibited BMSCs calcification.

\section{Discussion}

Previous studies have reported that miR-204 inhibited thyroid carcinoma cell proliferation and esophageal cancer cell invasion $(26,27)$. Additionally, downregulation of miR-204 enhanced osteogenesis in rat BMSCs (28). In the present study, it was observed that miR-204 agomir inhibited Runx2, ALP and BMP2 expression, and inhibited MSC calcification, the underlying mechanisms of these inhibitory effects were investigated.

The BMSCs isolated from rat bone marrow in the present study exhibited a $\mathrm{CD} 90^{+} / \mathrm{CD} 45^{-} / \mathrm{CD} 11 b^{-}$phenotype (Fig. 1A). Runx 2 regulates a series of cell cycle genes in endothelial cells, including cyclin-dependent kinase (CDK)4, CDK1 and cyclin B1 (29); additionally, reducing Runx 2 levels decreased breast tumor cell viability and inhibited cell migration (30). The loss of Runx 2 in chondrocytes impaired osteoprotegerin-receptor signaling and chondroclast development $(30,31)$. Increased bone turnover resulted in elevated serum ALP in females, and ALP levels are routinely used in the assessment of Paget's disease of bone $(32,33)$. High serum ALP levels were reported in patients with RA (18). The present findings revealed that the expression levels of Runx 2 and ALP increased with longer durations of BMSC culturing, suggesting that differentiation occurred in BMSCs when the cells were cultured for a long period of time. Additionally, miR-204 expression decreased in a time-dependent manner. Transfection with miR-204 agomir downregulated the expression of Runx 2 and ALP, suggesting that increased miR-204 levels inhibited the osteogenic differentiation of MSCs.

BMP2 has been reported to promote bone regeneration, and abnormal BMP2 levels result in bone diseases, as BMP2 

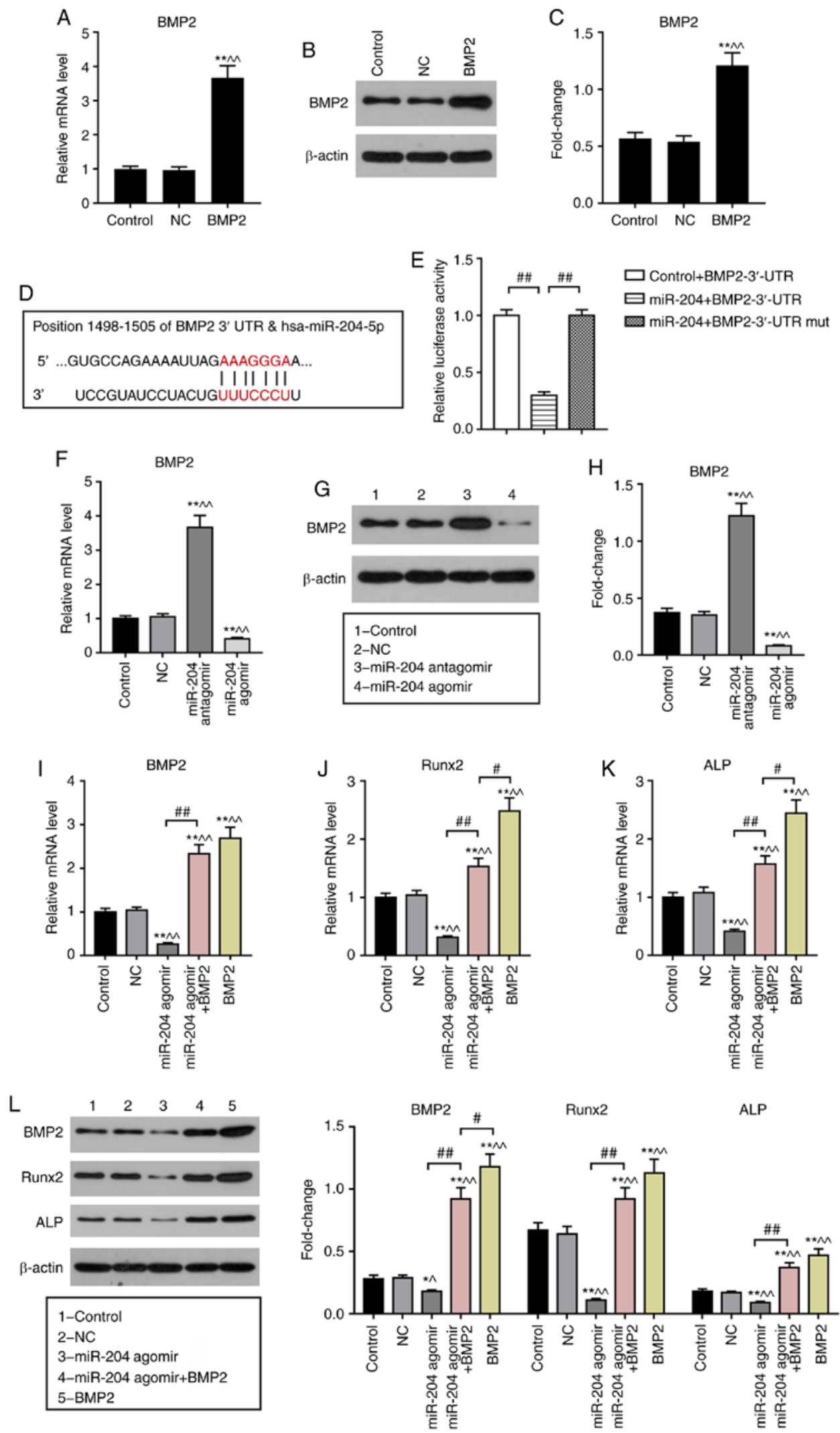

Figure 3. Effects of miR-204 agomir and BMP2 on the expression levels of Runx2, ALP and BMP2 in BMSCs. BMSCs were treated with NC, miR-204 agomir, miR-204 antagomir, BMP2 vector or miR-204 agomir + BMP2 vector for 3 days. $\beta$-actin and U6 were used as internal references. (A-C) BMP2 expression in BMSCs transfected with BMP2 vector as determined via RT-qPCR and western blot analyses. (D) Putative target genes and binding sites of miR-204 were predicted using TargetScan. (E) Interactions between miR-204 and the BMP2 3'-UTR were evaluated using dual-luciferase assays. (F) mRNA and $(\mathrm{G}$ and $\mathrm{H}$ ) protein expression of BMP2 in BMSCs transfected with miR-204 agomir or antagomir. (I-K) mRNA levels of BMP2, Runx2 and ALP in BMSCs transfected with miR-204 agomir and/or BMP2 vector, as determined via RT-qPCR analysis. (L) Protein levels of BMP2, Runx2 and ALP in BMSCs transfected with miR-204 agomir and/or BMP2 vector, as determined via western blotting. Data are presented as the mean \pm standard deviation and analyzed by ANOVA followed by Tukey-Kramer multiple comparison post hoc tests. ${ }^{*} \mathrm{P}<0.05,{ }^{* *} \mathrm{P}<0.01$ vs. control; ${ }^{\wedge} \mathrm{P}<0.05,{ }^{\wedge} \mathrm{P}<0.01$ vs. NC; ${ }^{\#} \mathrm{P}<0.05$, ${ }^{\# \#} \mathrm{P}<0.01$. BMSC, bone marrow mesenchymal stem cell; miRNA/miR, microRNA; BMP2, bone morphogenic protein 2; ALP, alkaline phosphatase; Runx2, Runt-related transcription factor 2; NC, negative control; 3'-UTR, 3'-untranslated region; mut, mutant. 


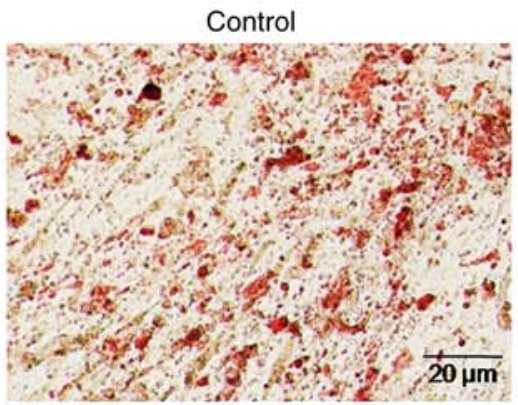

miR-204 agomir+BMP2

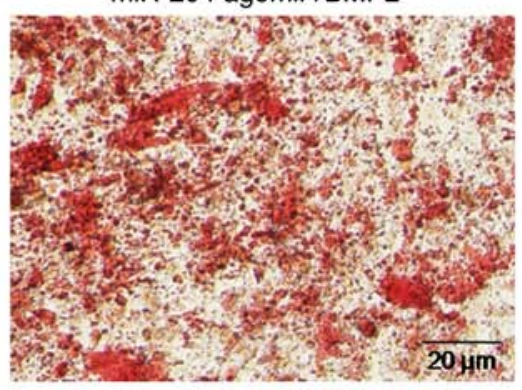

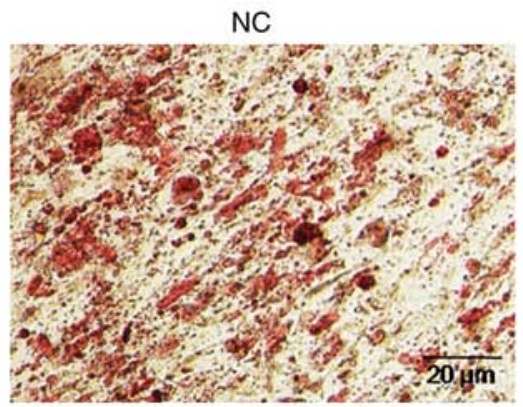

BMP2

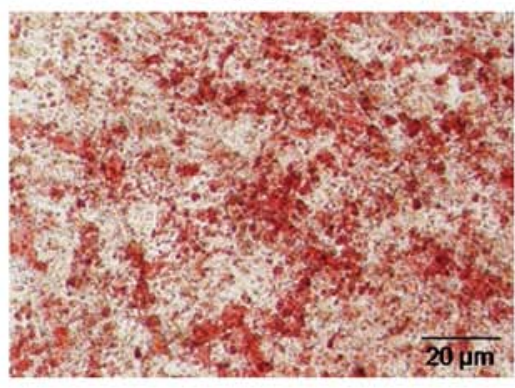

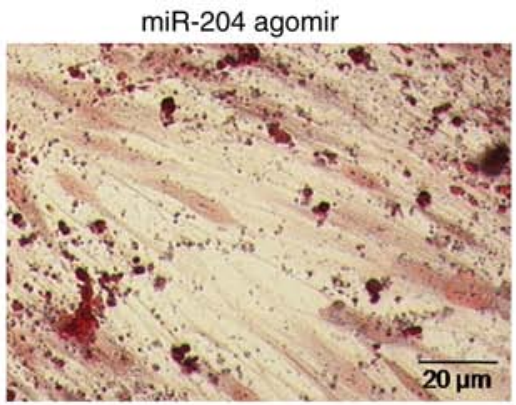

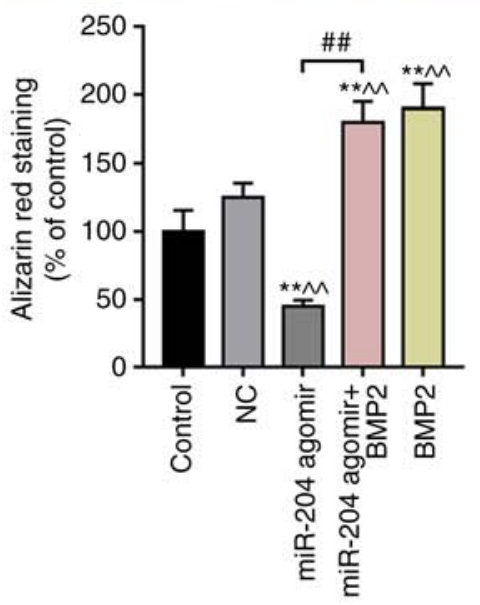

Figure 4. Effects of miR-204 agomir and BMP2 on calcification in BMSCs. BMSCs were seeded in 24-well plates, and treated with miR-204 agomir and/or BMP2 expression vector for 15 days; after $12 \mathrm{~h}$, the initial medium was replaced with medium supplemented with vitamin C and $\beta$-phosphoglycerol. BMSCs were dyed using alizarin red, and the acquired images were analyzed using ImageJ software. Data are presented as the mean \pm standard deviation and analyzed by ANOVA followed by Tukey-Kramer multiple comparison post hoc tests. ${ }^{* *} \mathrm{P}<0.01 \mathrm{vs}$. control; ${ }^{\wedge} \mathrm{P}<0.01 \mathrm{vs}$. NC; ${ }^{\# \#} \mathrm{P}<0.01$. BMSC, bone marrow mesenchymal stem cell; miR-204, microRNA-204; BMP2, bone morphogenic protein 2; NC, negative control.

promotes chondrogenesis, myogenesis, osteogenesis and bone mineral density (34,35). Additionally, BMP2-deficient embryos exhibited defects in cardiac development, which manifested as the abnormal development of the heart in the exocoelomic cavity (19). Reduced BMP2 in both embryonic and maternal tissues affected neural tube closure and body wall closure to varying degrees (22). In humans, there was a reduction in BMP2 expression in certain parts of unfractured bones compared with fractures in the process of healing, whereas BMP2 was expressed strongly in areas of healing; BMP2 levels gradually decreased as healing progressed (36-38). The results of the present study indicated that miR-204 agomir inhibited the expression of BMP2 in BMSCs. It was proposed that miR-204 agomir inhibited Runx 2 and ALP expression by regulating BMP2. To investigate this hypothesis, the expression levels of Runx 2 and ALP were determined in BMSCs transfected with a BMP2 overexpression vector. It was demonstrated that overexpression of BMP2 increased the levels of Runx 2 and ALP in BMSCs; however, miR-204 agomir downregulated the expression of Runx2 and ALP in BMP2-overexpressing BMSCs.

Skeletal mineralization requires connections between cellular activity and the extracellular environment; skeletal formation promotes the mineralization of the matrix (39). Numerous studies reported that the alizarin red stain assay can be used to evaluate the osteogenic capacity of BMSCs as determined by the extent of mineralization (40-43). The present findings suggested that BMP2 promoted the osteogenesis of BMSCs, and that miR-204 agomir reduced the osteogenic capacity of BMSCs by inhibiting BMP2; however, the present study did not include the use an animal model to further investigate whether miR-204 overexpression negatively affected osteogenic differentiation. Additionally, bioinformatics analysis was not conducted to identify other putative target genes of miR-204.

In conclusion, the results of the present study suggested that miR-204 upregulation inhibited the BMP2/Runx2/ALP signaling pathway by regulating BMP2. Additionally, the study provided preliminary evidence that miR-204 inhibited the differentiation of osteogenesis in BMSCs by targeting BMP2.

\section{Acknowledgements}

Not applicable.

\section{Funding}

No funding was received.

\section{Availability of data and materials}

The analyzed data sets used and/or anlayzed during the current study are available from the corresponding author on reasonable request. 


\section{Authors' contributions}

$\mathrm{XJ}$ and $\mathrm{ZZ}$ made substantial contributions to the conception and design of the present study. TP, GW, QX and GL were involved in data acquisition, analysis and interpretation. $\mathrm{XJ}$ and $\mathrm{ZZ}$ drafted the manuscript and critically revised it for important intellectual content. All authors gave final approval of the version to be published. All authors agreed to be accountable for all aspects of the work in ensuring that questions related to the accuracy or integrity of the work are appropriately investigated and resolved.

\section{Ethics approval and consent to participate}

The animal study was approved by the Institutional Animal Care and Use Committee of Yantai Yuhuangding Hospital.

\section{Patient consent for publication}

Not applicable.

\section{Competing interests}

The authors declare that they have no competing interests.

\section{References}

1. Kim BS, Kang HJ, Park JY and Lee J: Fucoidan promotes osteoblast differentiation via JNK- and ERK-dependent BMP2-Smad $1 / 5 / 8$ signaling in human mesenchymal stem cells. Exp Mol Med 47: e128, 2015.

2. Moon JS, Kim SH, Oh SH, Jeong YW, Kang JH, Park JC, Son HJ, Bae S, Park BI, Kim MS, et al: Relaxin augments BMP-2-induced osteoblast differentiation and bone formation. J Bone Miner Res 29: 1586-1596, 2014

3. Hu K and Olsen BR: Osteoblast-derived VEGF regulates osteoblast differentiation and bone formation during bone repair J Clin Invest 126: 509-526, 2016.

4. Li KL, Chen J, Li ZH, Zhao L and He YN: p53 negatively regulates the osteogenic differentiation of vascular smooth muscle cells in mice with chronic kidney disease. Cardiovasc J Afr 23 e1-e9, 2012.

5. Qian K, Xu H, Dai T and Shi K: Effects of Tanshinone IIA on osteogenic differentiation of mouse bone marrow mesenchymal stem cells. Naunyn Schmiedebergs Arch Pharmacol 388: $1201-1209,2015$

6. Papathanasopoulos A, Kouroupis D, Henshaw K, McGonagle D, Jones EA and Giannoudis PV: Effects of antithrombotic drugs fondaparinux and tinzaparin on in vitro proliferation and osteogenic and chondrogenic differentiation of bone-derived mesenchymal stem cells. J Orthop Res 29 1327-1335, 2011.

7. Peng S, Gao D, Gao C, Wei P, Niu M and Shuai C: MicroRNAs regulate signaling pathways in osteogenic differentiation of mesenchymal stem cells (Review). Mol Med Rep 14: 623-629, 2016

8. Jiang C, Sun J, Dai Y, Cao P, Zhang L, Peng S, Zhou Y, Li G, Tang $\mathrm{J}$ and Xiang J: HIF-1 A and C/EBPs transcriptionally regulate adipogenic differentiation of bone marrow-derived MSCs in hypoxia. Stem Cell Res Ther 6: 21, 2015.

9. Cui H, Yi Q, Feng J, Yang L and Tang L: Mechano growth factor E peptide regulates migration and differentiation of bone marrow mesenchymal stem cells. J Mol Endocrinol 52: 111-120, 2014.

10. Matsushita K, Itoh S, Ikeda S, Yamamoto Y, Yamauchi Y and Hayashi M: LIF/STAT3/SOCS3 signaling pathway in murine bone marrow stromal cells suppresses osteoblast differentiation. J Cell Biochem 115: 1262-1268, 2014.

11. Li T, Pan H and Li R: The dual regulatory role of miR-204 in cancer. Tumour Biol 37: 11667-11677, 2016.

12. Doench JG and Sharp PA: Specificity of microRNA target selection in translational repression. Genes Dev 18: 504-511, 2004.
13. Zhu X, Shen H, Yin X, Long L, Chen X, Feng F, Liu Y, Zhao P, Xu Y, Li M, et al: IL-6R/STAT3/miR-204 feedback loop contributes to cisplatin resistance of epithelial ovarian cancer cells. Oncotarget 8: 39154-39166, 2017.

14. Wu X, Zeng Y, Wu S, Zhong J, Wang Y and Xu J: MiR-204, down-regulated in retinoblastoma, regulates proliferation and invasion of human retinoblastoma cells by targeting CyclinD2 and MMP-9. FEBS Lett 589: 645-650, 2015.

15. Zhao J, Wang C, Song Y and Fang B: Arsenic trioxide and microRNA-204 display contrary effects on regulating adipogenic and osteogenic differentiation of mesenchymal stem cells in aplastic anemia. Acta Biochim Biophys Sin (Shanghai) 46: 885-893, 2014

16. Mansurabadi R, Abroun S, Hajifathali A, Asri A, Atashi A and Haghighi M: Expression of hsa-MIR-204, RUNX2, PPAR $\gamma$ and BCL2 in bone marrow derived mesenchymal stem cells from multiple myeloma patients and normal individuals. Cell J 19 (Suppl 1): S27-S36, 2017.

17. Groeneveld MC, Everts V and Beertsen W: Alkaline phosphatase activity in the periodontal ligament and gingiva of the rat molar: Its relation to cementum formation. J Dent Res 74: 1374-1381, 1995.

18. Nanke Y, Kotake S, Akama H and Kamatani N: Alkaline phosphatase in rheumatoid arthritis patients: Possible contribution of bone-type ALP to the raised activities of ALP in rheumatoid arthritis patients. Clin Rheumatol 21: 198-202, 2002.

19. Zhang H and Bradley A: Mice deficient for BMP2 are nonviable and have defects in amnion/chorion and cardiac development. Development 122: 2977-2986, 1996.

20. Ducy P and Karsenty G: The family of bone morphogenetic proteins. Kidney Int 57: 2207-2214, 2000.

21. Rosen V: BMP2 signaling in bone development and repair. Cytokine Growth Factor Rev 20: 475-480, 2009.

22. Singh AP, Castranio T, Scott G, Guo D, Harris MA, Ray M, Harris SE and Mishina Y: Influences of reduced expression of maternal bone morphogenetic protein 2 on mouse embryonic development. Sex Dev 2: 134-141, 2008.

23. Lamplot JD, Qin J, Nan G, Wang J, Liu X, Yin L, Tomal J, Li R, Shui $\mathrm{W}$, Zhang $\mathrm{H}$, et al: BMP9 signaling in stem cell differentiation and osteogenesis. Am J Stem Cells 2: 1-21, 2013.

24. Peng Y, Kang Q, Cheng H, Li X, Sun MH, Jiang W, Luu HH, Park JY, Haydon RC and He TC: Transcriptional characterization of bone morphogenetic proteins (BMPs)-mediated osteogenic signaling. J Cell Biochem 90: 1149-1165, 2003.

25. Livak KJ and Schmittgen TD: Analysis of relative gene expression data using real-time quantitative PCR and the 2(-Delta Delta C(T)) method. Methods 25: 402-408, 2001.

26. Liu L, Wang J, Li X, Ma J, Shi C, Zhu H, Xi Q, Zhang J, Zhao X and Gu M: MiR-204-5p suppresses cell proliferation by inhibiting IGFBP5 in papillary thyroid carcinoma. Biochem Biophys Res Commun 457: 621-626, 2015.

27. Sun Y, Yu X and Bai Q: miR-204 inhibits invasion and epithelial-mesenchymal transition by targeting FOXM1 in esophageal cancer. Int J Clin Exp Pathol 8: 12775-12783, 2015.

28. Shang G, Wang Y, Xu Y,Zhang S, Sun X, Guan H,Zhao X, Wang Y, Li Y and Zhao G: Long non-coding RNA TCONS 00041960 enhances osteogenesis and inhibits adipogenesis of rat bone marrow mesenchymal stem cell by targeting miR-204-5p and miR-125a-3p. J Cell Physiol 233: 6041-6051, 2018.

29. Pierce AD, Anglin IE, Vitolo MI, Mochin MT, Underwood KF Goldblum SE, Kommineni S and Passaniti A: Glucose-activated RUNX2 phosphorylation promotes endothelial cell proliferation and an angiogenic phenotype. J Cell Biochem 113: 282-292, 2012.

30. Taipaleenmaki $\mathrm{H}$, Browne $\mathrm{G}$, Akech J, Zustin J, van Wijnen AJ, Stein JL, Hesse E, Stein GS and Lian JB: Targeting of Runx 2 by miR-135 and miR-203 impairs progression of breast cancer and metastatic bone disease. Cancer Res 75: 1433-1444, 2015.

31. Chen H, Ghori-Javed FY, Rashid H, Adhami MD, Serra R, Gutierrez SE and Javed A: Runx2 regulates endochondral ossification through control of chondrocyte proliferation and differentiation. J Bone Miner Res 29: 2653-2665, 2014.

32. Mukaiyama K, Kamimura M, Uchiyama S, Ikegami S, Nakamura $\mathrm{Y}$ and Kato $\mathrm{H}$ : Elevation of serum alkaline phosphatase (ALP) level in postmenopausal women is caused by high bone turnover. Aging Clin Exp Res 27: 413-418, 2015.

33. Magnusson P, Davie MW and Sharp CA: Circulating and tissue-derived isoforms of bone alkaline phosphatase in Paget's disease of bone. Scand J Clin Lab Invest 70: 128-135, 2010 
34. Rogers MB, Shah TA and Shaikh NN: Turning bone morphogenetic protein 2 (BMP2) on and off in mesenchymal cells. J Cell Biochem 116: 2127-2138, 2015.

35. Deng M, Liu P, Xiao H, Zhang Y, Wang Y, Zhao J and Xu J: Improving the osteogenic efficacy of BMP2 with mechano growth factor by regulating the signaling events in BMP pathway. Cell Tissue Res 361: 723-731, 2015.

36. Kwong FN, Hoyland JA, Evans CH and Freemont AJ: Regional and cellular localisation of BMPs and their inhibitors' expression in human fractures. Int Orthop 33: 281-288, 2009.

37. Myers TJ, Longobardi L, Willcockson H, Temple JD, Tagliafierro L, Ye P, Li T, Esposito A, Moats-Staats BM and Spagnoli A: BMP2 regulation of CXCL12 cellular, temporal, and spatial expression is essential during fracture repair. J Bone Miner Res 30: 2014-2027, 2015.

38. Kwong FN, Hoyland JA, Freemont AJ and Evans CH: Altered relative expression of BMPs and BMP inhibitors in cartilaginous areas of human fractures progressing towards nonunion. J Orthop Res 27: 752-757, 2009.

39. Bensimon-Brito A, Cardeira J, Dionisio G, Huysseune A, Cancela ML and Witten PE: Revisiting in vivo staining with alizarin red S-a valuable approach to analyse zebrafish skeletal mineralization during development and regeneration. BMC Dev Biol 16: 2, 2016.
40. Ovchinnikov D: Alcian blue/alizarin red staining of cartilage and bone in mouse. Cold Spring Harb Protoc 2009: pdb.prot5170, 2009.

41. Nagy A, Gertsenstein M, Vintersten K and Behringer R: Alizarin red staining of post-natal bone in mouse. Cold Spring Harb Protoc 2009: pdb.prot5171, 2009.

42. Reynaud L and Jocteur-Monrozier A: Skeletal examination by alizarin staining. Methods Mol Biol 947: 201-213, 2013.

43. Bruneel B and Witten PE: Power and challenges of using zebrafish as a model for skeletal tissue imaging. Connect Tissue Res 56: 161-173, 2015.

(i)(3) This work is licensed under a Creative Commons Attribution-NonCommercial-NoDerivatives 4.0 International (CC BY-NC-ND 4.0) License. 